\title{
Accuracy of 3D position measurement of spherical objects with a holographic single camera setup
}

\author{
J. Guhathakurta, W. Li, S. Simon \\ Institute for Parallel and Distributed Systems (IPVS), Department of Parallel Systems \\ Universitätsstr. 38, 70569 Stuttgart, \\ Jajnabalkya.Guhathakurta@ipvs.uni-stuttgart.de
}

\begin{abstract}
In this paper, the accuracy with which a 3D position of a transparent spherical object can be measured, is investigated depending on the pixel size in the reconstructed image from a lens-less Mach-Zehnder holography setup. The ability to track 3D position of transparent spherical objects like bubbles or spray particles play an important role in mechanical process engineering. Since the 3D position of such objects are highly variable in time, standard imaging position measurement cannot be done as the objects frequently move in and out of focus and hence accurate position cannot be extracted from images. However, with lens-less holographic technique like the Mach-Zehnder holography, the 3D positon of an object can be measured without the need of focussing on the moving object. An experimental setup of the digital Mach-Zehnder off axis holography is presented in this paper and a quantitative analysis of the accuracy with which the 3D position of transparent spherical objects can be measured in the reconstructed image is investigated.
\end{abstract}

Keywords: Mach-Zehnder, Digital off axis Holography, Lens-less imaging, 3D position measurement.

\begin{abstract}
Introduction
The analysis of transparent spherical objects like bubbles (gas dispersed in liquid phase) or droplets (liquid dispersed in gaseous phase) play a crucial role in understanding their fluid dynamics in variety of applications like industrial chemical processes [1], fuel injection in combustion engines [2], agriculture [3], medicine etc. A large number of gas-liquid chemical processes e.g. hydrogenation, oxidation and chlorination reactions are performed in bubble chambers. The size, shape and velocities of these bubbles determine the parameters of the chemical reaction [4]. They are required to be measured, in order to control and optimize the chemical reaction itself. Similarly the characteristics of droplets are of prime interest in spray processes e.g. a reduction of fuel drop size in combustion engine generally leads to higher volumetric heat releases, easier lightup and lower concentration of pollutant emission while, in other applications like crop spraying, small droplets are avoided as they might drift too far away with winds [5].
\end{abstract}

In all the aforementioned applications measuring the characteristics of the bubbles or droplets becomes essential; however, due to their high and random dynamics, it becomes a challenging task to extract such information especially in real time. With the advances in high-speed cameras, real time shadowgraphy [6-7] has emerged as the most common tool to analyse the size and position of bubbles or droplets. It involves taking shadow images of objects, illuminated by a light source such as high power LED or lasers in different beam geometries (parallel beam, cone beam, light sheet etc.), at very high frame rates. Although this technique is simple and robust, it suffers from a few limitations.

The foremost limitation of shadowgraphy is the need of the object to be in focus. In case of both bubbles and droplets, they move frequently in and out of the focal plane and this limits their detection as they appear defocussed in the image and its surface position cannot be easily extracted. Secondly, if there are multiple objects present with overlapping positions along the direction of light, they appear as single objects in the shadow image and cannot be distinguished from one another. As this is often the case with bubbles or droplets, shadowgraphy might result in measuring wrong geometric dimensions and count of the objects in reality. Lastly, with this technique only the 2D position of the object can be determined in the focal plane. In order to determine the 3D position of object, shadowgraphy must be performed from at least two different optical axis (preferably perpendicular to each other). In 
certain applications [4] this is physically not possible due to space constrains.

The use of digital holography techniques like Mach-Zehnder off-axis holography eliminates the disadvantages of the shadowgraphy technique at the cost of more computation power for reconstructing the holograms. In this technique coherent light (lasers) are used to record the phase and intensity of the light transmitted through the object as interference patterns in the holograms. These holograms can be then reconstructed and digitally focused to get a sharp image and also the 3D position of the object. An application of this technique is already presented in [4], where the bubble position in Taylor-flow is extracted in reference to a point below it where the concentration measurement is done.

In this paper, a quantitative analysis of the accuracy of the digital Mach-Zehnder off-axis holography to determine 3D position of transparent spherical object, is presented. The uncertainty of the pixel size in reconstructed plane is calculated experimentally according to the Guide to the Expression of Uncertainty in Measurement (GUM) [8].

\section{Digital Mach-Zehnder off-axis holography}

In this holographic technique a laser beam is divided in to two equal parts, from which only one of them is made to pass through the object. Both the beams are then directed and recombined to capture the interference pattern with a digital image sensor. This interference image, known as the hologram, encodes the intensity as well as phase of the object as intensity profiles. A simplified diagram of this holographic technique is shown in Fig. 1.

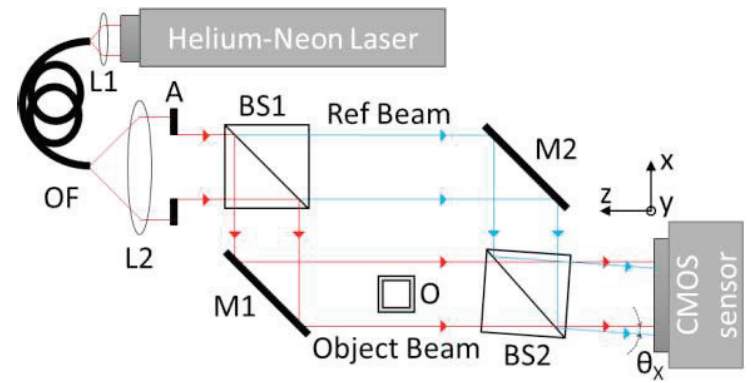

Fig. 1: Digital Mach-Zehnder off-axis holography setup.

A Helium-Neon (HeNe) laser $(\lambda=633 \mathrm{~nm})$ is chosen as the laser source due to its stable performance and high coherence length. The parallel beam of this laser is focused into a monomode glass fibre, OF with 4 um core diameter using the coupler L1. On the other end of the fibre, the diverging beam is collimated into a parallel beam of $10.8 \mathrm{~mm}$ diameter $\left(1 / \mathrm{e}^{2}\right.$ level of the Gaussian intensity distribution) using the fibre collimator L2. The ends of this fibre used has a cut angle of $8^{\circ}$ to prevent reflection back into the laser. The small core diameter also acts as a pinhole of a spatial filter to have a good quality collimated beam. This collimated beam is cropped by a variable aperture $A$ and then is split into two perpendicular beams by the beam splitter BS1 with intensity ratio of 50:50. The beam including the object $O$ in its optical path is referred to as the object beam and the other one as the reference beam. Both, the object beam and reference beam, are directed by mirrors M1 and $\mathrm{M} 2$ respectively to the second beam splitter BS2. The angles, at which they meet $\left(\theta_{x} \& \theta_{y}\right)$ at the camera sensor, determines the fringe pattern of the hologram and also the position of the twin image. The hologram is recorded using a custom real-time process analysis system developed at IPVS. This system features a high speed 1696 pixels $\times 1710$ pixels CMOS sensor with a pixel size of $8 \mu \mathrm{m}$. It allows real time imaging with an exposure time as low as 70 ns and with frame rates up to $485 \mathrm{fps}$ at full resolution. The short exposure time makes the holographic setup resistant to vibrations and mode hopping problems of the laser. Due to the huge volume of data generated by the sensor it is connected to a powerful field- programmable gate array (FPGA) to process the data and also apply image processing algorithms like holographic reconstruction directly in the realtime process analysis system. The Cartesian coordinate system in our experiment is depicted in Fig. 1 with the $x-y$ plane along the CMOS sensor plane and $z$ axis perpendicular to the plane of the CMOS sensor. The intensity distribution in the hologram plane is given by:

$$
h(x, y, 0)=|O(x, y, 0)+R(x, y, 0)|^{2}
$$

where, $O(x, y, 0)$ and $R(x, y, 0)$ represents the complex wave of the object and reference beam in the hologram plane, respectively (for simplicity they are henceforth referred to as just $O$ and $R$ ). The above equation can be expanded as:

$$
\begin{aligned}
h & =(O+R)(O+R)^{*} \\
& =|O|^{2}+|R|^{2}+O R^{*}+R O^{*}
\end{aligned}
$$

In order to reconstruct the hologram it is illuminated with the same reference wave as the one it was captured with. The reconstructed image intensity is given by:

$$
\begin{aligned}
I=h R & =\left(|O|^{2}+|R|^{2}\right) R+O R^{*} R+R O^{*} R \\
& =\left(|O|^{2}+|R|^{2}\right) R+O|R|^{2}+O^{*} R^{2}
\end{aligned}
$$

The reconstructed image has three components as shown in the above equation. The first term 
represents the undiffracted wave passing the hologram and is the zero order DC term which only influences the overall brightness of the hologram. The second term represents the reconstructed object wave multiplied by a constant factor which forms the virtual image and the third term represents a defocussed real image of the object commonly referred to as the twin image. Out of these three term only the second term contains useful information. To remove the zero order component a 3 pixel $\times 3$ pixel averaging filter is applied to the hologram and the result is subtracted from the original hologram. On reconstructing the resulting hologram the DC component is removed but the twin image remains. The position of the twin image depends on the angles at which the object and reference beam meet at the hologram plane $\left(\theta_{x} \& \theta_{y}\right)$. In case of in-line holography [9], $\theta_{x}=\theta_{y}=0^{\circ}$ and the twin image overlaps with the virtual image of the object but in case of off-axis holography [10] the two angles $\left(\theta_{x} \& \theta_{y}\right)$ allow the spatial separation of the twin image from the virtual image in the reconstructed plane. However, careful attention must be paid to the adjustment of $\theta_{x} \& \theta_{y}$, which must not exceed a maximum value $\theta_{\max }$ for which the carrier frequency of the hologram is equal to the Nyquist frequency of the sensor [11],

$$
\theta_{x} \& \theta_{y} \leq \theta_{\max }=\arcsin \left(\frac{\lambda}{2 \Delta_{p i x}}\right)
$$

Where, $\lambda$ is the wavelength and $\Delta_{p i x}$ is the pixel size of the CMOS sensor. For our setup $\theta_{\max }=$ $2.26^{\circ}$. The twin image is thus separated spatially by adjusting $\mathrm{M} 1$ and $\mathrm{M} 2$ along with the rotation of BS2 as shown Fig. 1.

\section{Digital Reconstruction}

In digital holography the captured hologram can be reconstructed numerically at different planes. The most commonly used reconstruction algorithms are Fresnel transform, angular spectrum and convolution algorithm [12]. All of these algorithms are based on fast-Fourier-transform (FFT) which are highly resource consuming when implemented on FPGAs. The Fresnel transform approach involves the computation of only one FFT while the angular spectrum and convolution algorithm requires the computation of two and three FFT respectively. As we intend to apply our system in real time on a FPGA with limited resources, we choose Fresnel transform as our digital reconstruction algorithm to demonstrate the accuracy of our measuring technique. The reconstructed image $U(x, y, z)$, at a distance of $z$, obtained by Fresnel transform is given by the formula:

$$
\begin{aligned}
& U(x, y, z) \\
& =\frac{e^{i k z}}{i \lambda z} e^{\frac{i k}{2 z}\left(x^{2}+y^{2}\right)} F\left\{h\left(x_{0}, y_{0}\right) e^{-\frac{i k}{2 z}\left(x_{0}^{2}+y_{0}^{2}\right)}\right\}
\end{aligned}
$$

where, $h\left(x_{0}, y_{0}\right)$ is the hologram, $\lambda$ is the wavelength, $k$ is the wavenumber and $F\{\}$ denotes the Fourier transform which is implemented digitally by FFT. From the computation perspective it involves three steps. First, multiplying the intensities of the hologram with a factor. Second, computing a 2D FFT of the product and lastly multiplying it with another factor. The size of the pixels in the reconstructed image at a distance $z$ is given in literature by:

$$
\Delta x=\Delta y=\frac{\lambda \times z}{\Delta_{p i x} \times N}
$$

where, $\Delta_{\text {pix }}$ is the pixel size of the camera and $N$ is the number of pixels in a row or column. Since the reconstructed pixel size is directly proportional to $z$, the closer the object is to the camera better is the resolution. However, as $Z$ decreases the field of view also decreases and the spatial separation of the twin image becomes difficult. Hence, a trade-off has to be achieved between resolution, measured area and the spatial separation of the twin image is.

The reconstruction distance $z$ is given as an input parameter to the reconstruction algorithm. In order to find the position of the object along the $z$ axis, reconstruction is done over a range of values for $z$ and the distance at which the sharpest image is obtained is considered as the object depth. In order to have an automatic measure of the sharpest image digital autofocussing algorithm is used. Out of the various auto-focus evaluation functions like variance of grey function, weighted Fourier spectral function and standard deviation correlation function etc., the variance of grey level shows the most sensitivity to detecting sharp images [13] and is hence chosen for determining the depth of the object. The measure of image sharpness by this method is given by:

$$
\operatorname{Var}=\frac{1}{N^{2}} \sum_{m=1}^{N} \sum_{n=1}^{N}[I(m, n)-\bar{I}]^{2}
$$

where, $I$ denotes an image of $N \times N$ pixels and

$$
\bar{I}=\frac{1}{N^{2}} \sum_{m=1}^{N} \sum_{n=1}^{N} I(m, n) .
$$


The distance $z$ at which the reconstructed image has the maximum value of $\operatorname{Var}$ gives the depth of the object.

\section{Experimental results}

The experimental setup of the digital MachZehnder off axis holography is shown in Fig. 2. The components are labelled in comparison to the Fig. 1. The object $O$ in Fig. 2 is an USAF target with elements from group 2 to group 7 . This USAF target has been mounted on a 3 axis linear stage with precision steps of 10 um. For the first experiments a red laser diode $(\lambda=$ $658 \mathrm{~nm}$ ) at $10 \mathrm{~mW}$ output power was used as the coherent source. The position of mirrors M1 and M2 along with the rotation of BS2 were adjusted so as to have an optimum spatial separation of the twin image as explained in the previous section.

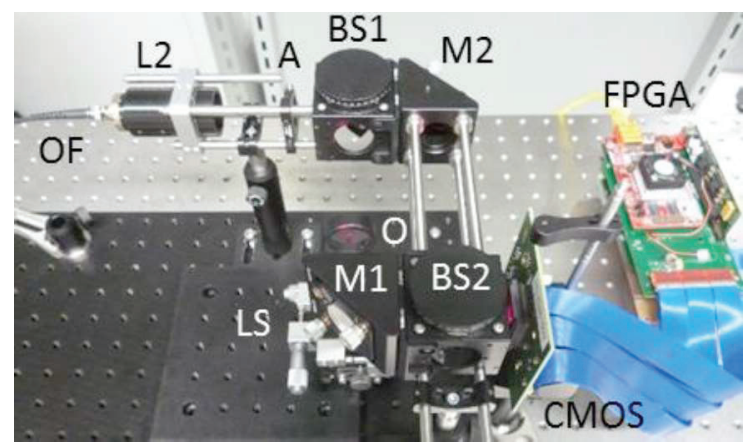

Fig. 2: Experimental setup

The hologram of the USAF target was captured by the CMOS sensor with an exposure time of 200 ns and is depicted in Fig. 3. The combination of laser output power and exposure time of the CMOS sensor has been chosen in such a way that a good contrasting hologram is recorded.

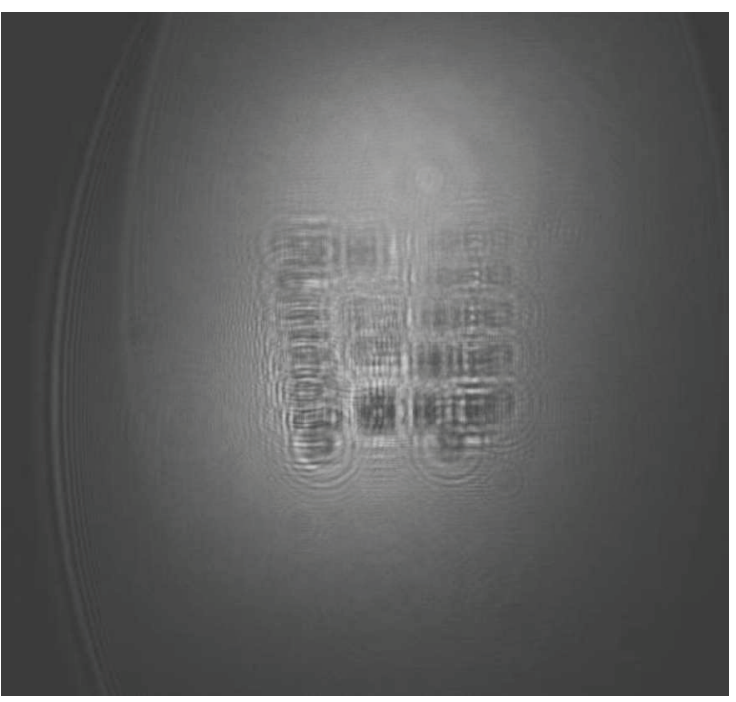

Fig. 3: Hologram of the USAF target
Before reconstruction the hologram was preprocessed to remove the DC term as explained in the previous section and then a Fresnel's transform was applied to obtain a reconstruction image (Fig. 4). As discussed before, it contains the sharp virtual image and an unfocussed real twin image; however, due to the off-axis arrangement they are spatially separated in the reconstruction plane.

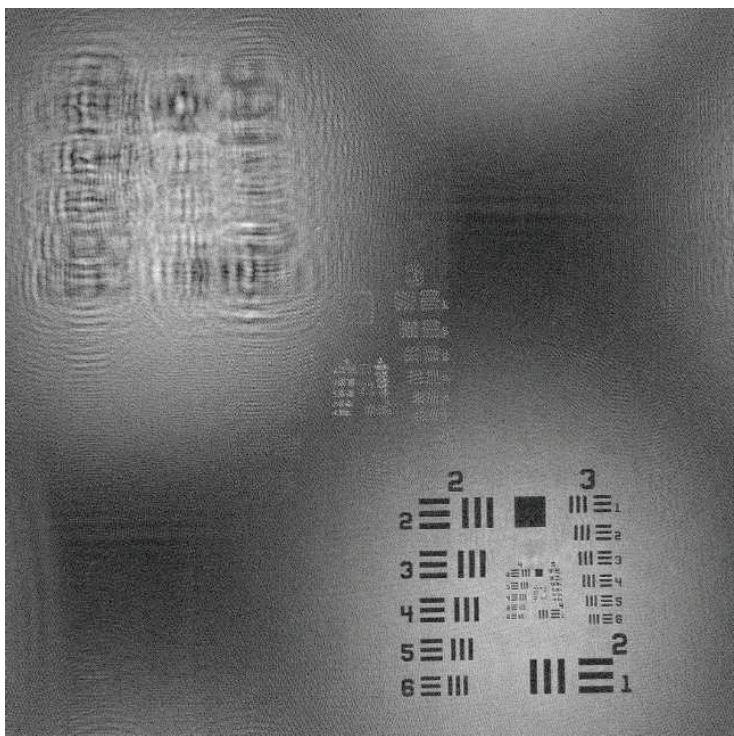

Fig. 4: $\quad$ Reconstructed hologram at $z=161.65$ $\mathrm{mm}$

For automatic depth detection the auto-focus variance measure was calculated only in the region of the virtual image and plotted for reconstruction images with different $z$ as shown in Fig.5.

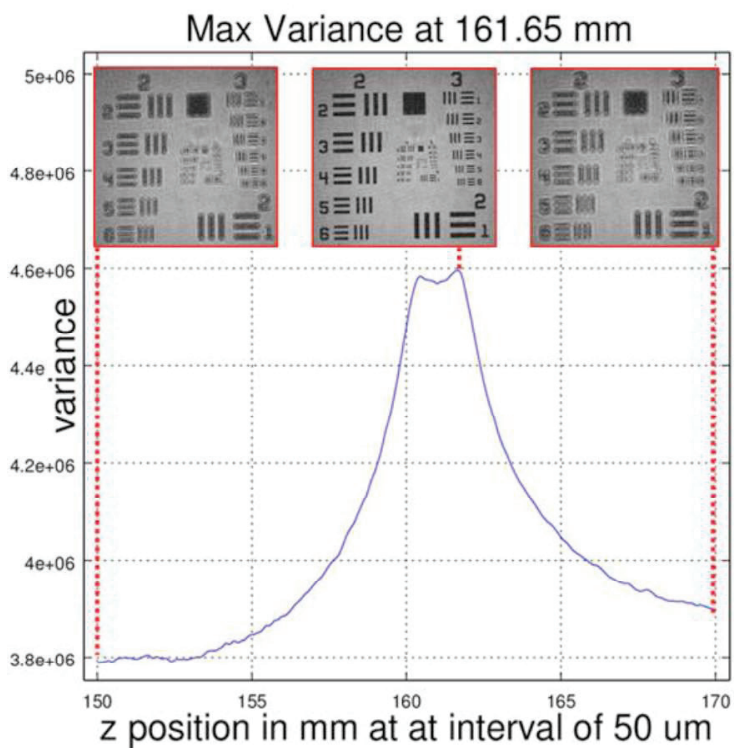

Fig.5: Auto-focus variance (Var) plot for reconstructed images at different $\mathrm{z}$ positions 
The $z$ position where the value of $\mathrm{Var}$ is maximum gives the depth of the object. In the above case the maximum is at $z$ position 161.65 $\mathrm{mm}$. The two sub peaks in Fig. 1 correspond to the two surface of the USAF target which has a substrate thickness of $1.5 \mathrm{~mm}$ and the line pair patterns (120 um thick) are on the back surface of the substrate. Hence, the second peak corresponds to USAF pattern and has a higher $\operatorname{Var}$.

For the accuracy of position measurement of transparent spherical objects in $x$ and $y$ direction, the size of the pixels in the reconstructed plane must be experimentally derived $\left(\Delta x_{e} \& \Delta y_{e}\right)$ according to GUM and also compared to the value $(\Delta x \& \Delta y)$. For this a custom target from Thorlabs is used which contains concentric circles of known sizes and having a structure tolerance of $0.5 \mathrm{um}$. Multiple holograms are captured with this target and the reconstructed images are analysed to determine $\Delta x_{e} \& \Delta y_{e}$. One such hologram and its reconstruction $(z=)$ is shown in Fig. 6 .
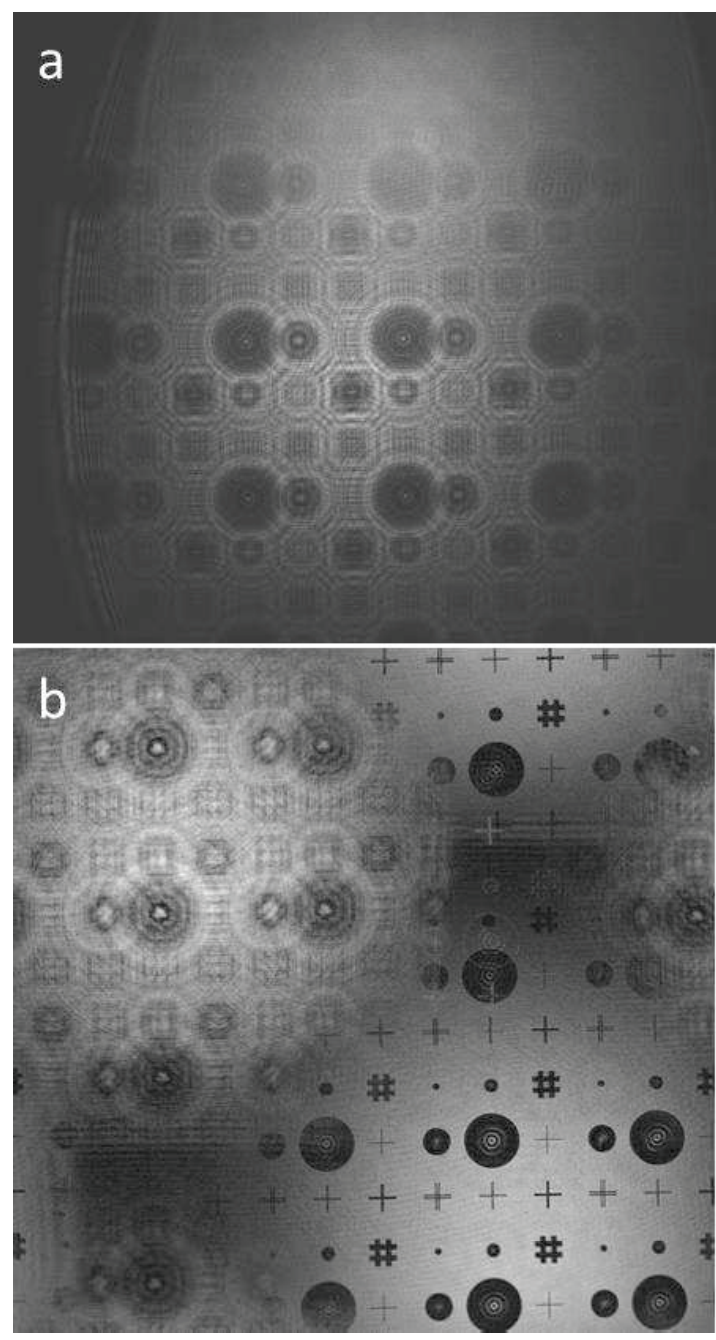

Fig. 6: (a) Hologram (b) Reconstruction
For the accuracy of the position measurement in $z$ direction, the target was moved in fixed steps of 50 um along the $z$ axis with the help of the linear stages (LS) in Fig. 2. At each step ten holograms were captured and the object positions, obtained from the auto-focusing after reconstruction, are analysed to see if the step sizes were resolvable. For this part of the experiment the HeNe laser $(\lambda=633 \mathrm{~nm})$ was used for better wavelength stability.

\section{Accuracy analysis}

From direct analysis of resolution from the reconstructed image of the USAF target in Fig. 4, the smallest line pairs which are distinguishable in both horizontal and vertical direction are of group 5 element 3 . According to the USAF resolution charts this corresponds to a system resolution of $40.3 \mathrm{lp} / \mathrm{mm}$.

The accuracy of position measurement depends on the pixel size in the reconstruction plane. The pixel size in both directions $\left(\Delta x_{e} \& \Delta y_{e}\right)$ in the reconstructed plane is calculated experimentally by counting the number of pixel corresponding to a fixed known length. The fixed known length we have chosen in our case are the largest circle in the reconstructed image Fig. 6 (b) which are known, from datasheet of the fabricated target, to have a diameter $D=1000 \mu \mathrm{m}$ and an uncertainty $u(D)=1 \mu \mathrm{m}$. In order to count the number of pixels, $p$, corresponding to this circle the intensity profile of each row of the region containing the circle is plotted, a thresholding value is determined using Otsu's method [14], and the pixels between the left and right edge of the circle is counted. The row having the maximum count of pixel between the left and right edge of the circle corresponds to the diameter $D$ of the circle. An intensity profile of the diameter row is plotted in Fig.7.

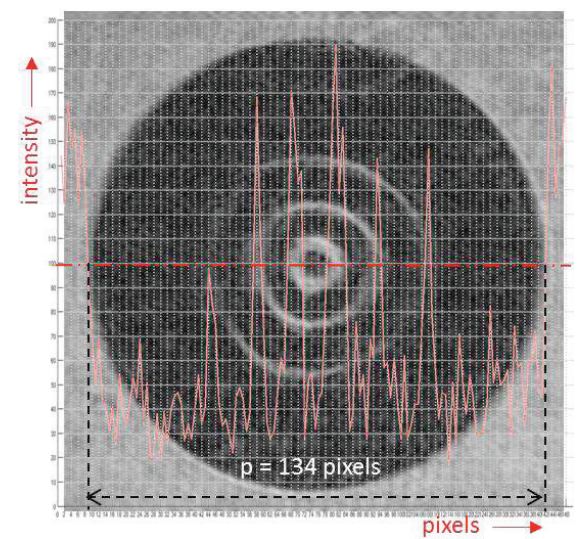

Fig.7: Intensity profile of the row corresponding to the diameter of the circle 
The pixel count along the horizontal axis $\left(0^{\circ}\right)$ was found to be 134 . The diameter is also measured along three other axes $45^{\circ}, 90^{\circ}$ and $135^{\circ}$. For this, the reconstructed image is rotated with the respective angle and the number of pixels along the diameter is obtained as explained previously. The angles were chosen in such a way so that interpolation errors by image rotation are minimal. The pixel count along the diameter at the four different angles are shown in Tab.1.

\section{Tab.1: $\quad$ Pixel count along the diameter}

\begin{tabular}{|c|c|c|c|c|}
\hline angles & $0^{\circ}$ & $45^{\circ}$ & $90^{\circ}$ & $135^{\circ}$ \\
\hline$p$ & 133 & 134 & 134 & 135 \\
\hline
\end{tabular}

According to GUM type A the uncertainty of the average pixel count $u(\bar{p})$ along the diameter is given by:

$$
u(\bar{p})=\frac{s(p)}{\sqrt{i}}
$$

where, $s(p)$ is the standard deviation of the values of $p$ and $i$ is the number of measurement. In our case $s(p)=0.8165$ and $i=4$. Therefore, $u(\bar{p})=0.4083 \mu \mathrm{m}$. The average value of pixel count along the diameter is $\bar{p}=134$.

The experimental pixel size $\left(\Delta x_{e} \& \Delta y_{e}\right)$ in the reconstruction plane is given by:

$$
\Delta x_{e}=\Delta y_{e}=f(D, p)=\frac{D}{\bar{p}}=7.463 \mu \mathrm{m}
$$

According to propagation of uncertainty in GUM the uncertainty of $\Delta x_{e} \& \Delta y_{e}$ is given by:

$$
u\left(\Delta x_{e}\right)=u\left(\Delta y_{e}\right)=\sqrt{c_{1}^{2} \cdot u^{2}(D)+c_{2}^{2} \cdot u^{2}(\bar{p})}
$$

where,

$$
c_{1}=\frac{\partial f}{\partial D}=\frac{1}{\bar{p}} \quad \& \quad c_{2}=\frac{\partial f}{\partial \bar{p}}=-\frac{D}{\bar{p}^{2}}
$$

From the above two formulas:

$$
u\left(\Delta x_{e}\right)=u\left(\Delta y_{e}\right)=0.023932 \mu m
$$

Therefore the accuracy of the reconstructed pixel size is:

$$
\Delta x_{e}=\Delta y_{e}=7.463 \mu \mathrm{m} \pm 0.023932 \mu \mathrm{m}
$$

Whereas, the reconstructed pixel size $(\Delta x \& \Delta y)$ calculated from the equation discussed in the section of digital reconstruction is:

$$
\Delta x=\Delta y=7.579 \mu m
$$

where, $\lambda=658 \mathrm{~nm}, z=156.5 \mathrm{~mm}, \Delta_{\text {pix }}=$ $8 \mu \mathrm{m}$ and $N=1696$. It can be observed that these values are in close correspondence to the experimental values. The minor variation might be due to instabilities in the laser wavelength.

For the accuracy of position measurement along $z$ direction, the target is moved along the z-axis closer to the CMOS sensor in steps of 50 um and at each step ten holograms were captured. The HeNe laser with $\lambda=633 \mathrm{~nm}$ was used as the coherent source in this case. For each hologram, reconstruction and autofocussing was done to determine the object position along the $z$ axis. Each cell in Tab.2 corresponds to one such object position, i.e. each column corresponds to 10 reconstruction

\begin{tabular}{|c|c|c|c|c|c|}
\hline Pos. & $0 \mathrm{um}$ & 50 um & 100 um & 150 um & 200 um \\
\hline 1 & 167276 & 167295 & 167198 & 167158 & 167077 \\
\hline 2 & 77308 & 167282 & 167209 & 167154 & 167057 \\
\hline 3 & 57293 & 167261 & 167191 & 167123 & 67104 \\
\hline 4 & 57284 & 167275 & 167195 & 167146 & 167097 \\
\hline 5 & 57289 & 16722 & 167230 & 167182 & 167086 \\
\hline 6 & 57263 & 167293 & 167232 & 167135 & 167103 \\
\hline 7 & 57295 & 167294 & 167222 & 167132 & 167045 \\
\hline 8 & 57388 & 167245 & 167264 & 167175 & 167057 \\
\hline 9 & 167299 & 167226 & 167221 & 167142 & 167086 \\
\hline 10 & 167287 & 167259 & 167235 & 167117 & 167114 \\
\hline Mean & 167298 & 167265 & 167220 & 167146 & 167083 \\
\hline$\Delta z_{e}$ & - & 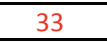 & 45 & 74 & 63 \\
\hline Pos. & 0 um & um & m & Im & 450 um \\
\hline 1 & 167025 & 166992 & 166965 & 84 & 166835 \\
\hline 2 & 167028 & 166978 & & & 166830 \\
\hline 3 & 167036 & 167019 & 166981 & 66951 & 166846 \\
\hline 4 & 167029 & 167009 & 166999 & 166930 & 166817 \\
\hline 5 & 167004 & 167006 & 10 & 166951 & 166836 \\
\hline 6 & 57024 & 167005 & 166965 & 166 & 166927 \\
\hline 7 & 167024 & 167017 & 166991 & 166972 & 166887 \\
\hline 8 & 167009 & 166991 & 167004 & 166980 & 166901 \\
\hline 9 & 167033 & 167026 & 166996 & 166994 & 166919 \\
\hline 10 & 167042 & 167021 & 166974 & 167009 & 166858 \\
\hline Mean & 167025 & 167006 & 166989 & 166971 & 166866 \\
\hline$\Delta z_{e}$ & 58 & 19 & 18 & 17 & 105 \\
\hline
\end{tabular}
distances at every step and the last row of the table gives the calculated step size, $\Delta z_{e}$ with respect to the previous step.

Tab.2: $\quad$ Reconstructed object position along zaxis in steps of 50 um (all dimensions in um)

It was observed that any step sizes below 30 um were not resolvable. For 50 um steps, the movement of the object was resolvable and the mean of the calculated step sizes is:

$$
\overline{\Delta z_{e}}=48 \mu \mathrm{m}
$$

The uncertainty of the mean step size was calculated according to GUM type A measurement:

$$
u\left(\overline{\Delta z_{e}}\right)=\frac{s\left(\Delta z_{e}\right)}{\sqrt{i}}=9.478 \mu m .
$$


where, $s\left(\Delta z_{e}\right)$ is the standard deviation of $\Delta z_{e}$ and $i$ is the number of step measurements which in our case is nine.

\section{Conclusion}

In this paper, the accuracy with which a 3D position of transparent spherical objects can be extracted has been investigated depending on the pixel size in the reconstructed image obtained from digital Mach-Zehnder off-axis holography. The Mach-Zehnder approach not only eliminates the focussing problem in determining the position of such objects by shadowgraphy but also gives a 3D position with a certain accuracy.

For the position accuracy analysis, the uncertainty of the experimentally calculated pixel size in the $x$ and $y$ direction was derived according to the standards of GUM. At a distance of around $156.5 \mathrm{~mm}$ the experimental pixel size was found to be $7.463 \mu \mathrm{m} \pm$ $0.023932 \mu \mathrm{m}$ in the presented setup. For the accuracy of position measurement along $z$ direction, it was found that step distances below 30 um was not resolvable by this measurement technique and the uncertainty of position measurement along $z$ direction at steps of 50 um was found to be around $10 \mathrm{um}$.

\section{Acknowledgements}

We gratefully thank the German Research Foundation within the priority program 1740 "Reactive Bubbly Flows", part RI 2512/1-1.

\section{References}

[1] J. Magnaudet and I. Eames, "The Motion of High-Reynolds-Number Bubbles in Inhomogeneous Flows," Annual Review of Fluid Mechanics, vol. 32, no. 1, 659-708 (2000); doi: 10.1146/annurev.fluid.32.1.659

[2] J. Swithenbank, . J. Beer, D. Taylor, . D. Abbot and G. Mccreath, "A laser diagnostic technique for the measurement of droplet and particle size distribution," in American Institute of Aeronautics and Astronautics, 14th Aerospace Sciences Meeting, Washington,DC (1976); doi:10.2514/6.1976-69

[3] B. Hijazi, T. Decourselle, S. Minov, D. Nuyttens, F. Cointault, J. Pieters and J. Vangeyte, "The Use of High-Speed Imaging Systems for Applications in Precision Agriculture," in New Technologies - Trends, Innovations and Research, InTech (2012); doi: 10.5772/38778

[4] D. Schurr, J. Guhathakurta, Y. Baroud, S. Simon, G. Rinke and R. Dittmeyer, "Local concentration measurements in the wake of bubbles based on in-situ Raman spectroscopy and statistical analysis," in 9th International
Conference on Multiphase Flow, Firenze, Italy, (2016)

[5] A. H. Lefebvre, Atomization and Sprays, Taylor \& Francis (1989)

[6] S. Thoroddsen, T. Etoh and K. Takehara, "High-Speed Imaging of Drops and Bubbles," Annual Review of Fluid Mechanics, vol. 40, no. 1, 257-285 (2008); doi:10.1146/annurev.fluid.40.111406.102215

[7] M. Klaiber, J. Guhathakurta, W. Li, Z. Wang, A. Lampa, H. Li, S. Simon, M. Sommerfeld and U. Fritsching, "A High-Speed Process Monitoring System to Detect and Analyze Filaments and Droplet Collisions in Spray Processes in Real Time," in 13th Triennial International Conference on Liquid Atomization and Spray Systems, Tainan (2015)

[8] "Evaluation of Measurement Data-Guide for the Expression of Uncertainty in Measurement," BIPM, IEC and IFCC, ILAC and IUPAC, IUPAP (2008)

[9] D. Gabor, "A New Microscopic Principle," Nature, vol. 161, pp. 777-778 (1948); doi:10.1038/161777a0

[10] E. Leith and J. Upatnieks, "Microscopy by Wavefront Reconstruction," Journal of the Optical Society of America, vol. 55, no. 5, pp. 569-570 (1965); doi: 10.1364/JOSA.55.000981

[11] E. Cuche, P. Marquet and C. Depeursinge, "Spatial filtering for zero-order and twin-image elimination in digital off-axis holography," Applied Optics, vol. 39, no. 23, 4070-4075, (2000); doi: 10.1364/AO.39.004070

[12] W. Huaying, Z. Baoqun and L. Wei, "Comparison of the reconstruction algorithms in digital micro-holography," in IEEE International Conference on Intelligent Computing and Intelligent Systems, Shanghai (2009); doi: 10.1109/ICICISYS.2009.5358270

[13] H. Wang, A. Qin and M. Huang, "Autofocus Method for Digital Holographic Reconstruction of Microscopic Object," in Symposium on Photonics and Optoelectronics, Wuhan (2009); doi: 10.1109/SOPO.2009.5230170

[14] N. Otsu, "A Threshold Selection Method from Gray-Level Histograms," IEEE Transactions on Systems, Man, and Cybernetics, vol. 9, no. 1, 62-66 (1979); doi: 10.1109/TSMC.1979.4310076 